\title{
Introducción.
}

\section{Los nuevos rumbos del Coneicc}

\author{
María Concepción Lara Mireles y \\ Ángel Manuel Ortiz Marín
}

La tarea de introducir un texto implica variadas condiciones. Por una parte, el obligado paso de ofrecer al lector un panorama de la obra que tiene en sus manos y darle pistas de aquellos temas y autores que puedan ser de su interés, a la vez que plasmar el sentir de quienes participan en este esfuerzo editorial.

Fiel a su tradición de ser el escenario que durante 16 de los últimos 32 años que tiene de existencia el Consejo Nacional para la Enseñanza y la Investigación de las Ciencias de la Comunicación (Coneicc), el Anuario refleja puntualmente la producción de los académicos e investigadores de las instituciones afiliadas a este organismo.

Esta publicación científica se constituye, en buena medida, en el referente de las preocupaciones y ocupaciones intelectuales de los estudiosos de la disciplina por abordar los temas que atañen a la realidad nacional y a su vez, en un foro de expresión de la producción del campo de las ciencias de la comunicación, en sus diversas facetas y escenarios.

La introducción de la edición XVI del Anuario de Investigación de la Comunicación Coneicc agrega una condición adicional y un objetivo particular: explicar el proyecto académico que el nuevo comité directivo impulsará por los próximos tres años (2009-2012). De ahí la pertinencia de ofrecer una exposición de las principales líneas de acción que se han planteado y que requerirán del concurso de los miembros institucionales y a título personal que integran el vasto directorio del Consejo.

La introducción de esta obra se ha divido en dos partes. La primera, denominada "El plan", referirá esta condición ya expresada. Y la segunda, "La obra", describirá la estructura del contenido, dando pie a la exposición de una sucinta reseña de los temas abordados por los autores, por orden alfabético, de esta edición XVI del Anuario.

\section{EL PLAN}

La razón de ser del Consejo Nacional para la Enseñanza y la Investigación de las Ciencias de la Comunicación es la de propiciar la comunicación 
entre profesores, investigadores e instituciones de ciencias de la comunicación para buscar juntos la comprensión de los problemas sociales y educativos de la realidad nacional, y tratar de encontrar soluciones a los mismos, a través de un trabajo colegiado y de redes interinstitucionales, es decir, aprovechando sinérgicamente los recursos humanos, metodológicos y materiales de los miembros asociados. El fomento de la investigación, la enseñanza y la extensión de las ciencias de la comunicación constituye el centro de la tarea colegiada y de intervención social del Consejo.

En consonancia con estos objetivos identitarios, el Comité Coordinador 2009-2012, cuerpo colegiado representativo de la pluralidad y dinamismo del Consejo, ha elaborado un plan de acción que gira en torno a tres ejes programáticos, íntimamente relacionados:

- Buscar una mayor presencia e incidencia social del Consejo.

- Fomentar el trabajo en redes regionales y nacionales para promover la investigación y la consolidación de los programas académicos de ciencias de la comunicación.

- Incursionar en nuevas modalidades científicas y académicas de desarrollo.

Por motivos de espacio los bosquejaremos solamente, y lo haremos en una forma interrelacionada y articulada, que es así como se da la dinámica del discurso y del quehacer complejo de la academia. Nuestro intento se sitúa en la perspectiva del fomento de redes de comunicación regionales y nacionales, como el referente obligado de un Anuario de Investigación que intenta horizontalizar el conocimiento, hacer fluir información y hallazgos basados en diferentes enfoques, y vincular diferentes objetos de estudio en un diálogo interdisciplinario.

$\mathrm{Y}$, precisamente, la redificación que queremos impulsar desde el Coneicc, está basada en procesos de construcción común, en la priorización del trabajo colegiado de las vocalías, en el seguimiento de sus proyectos regionales, en el desarrollo de programas de capacitación y actualización docente y formación del estudiantado, en el apuntalamiento de la formación de sus jóvenes investigadores, y en el impulso de la publicación de la producción académica de sus profesores e investigadores.

Al Anuario de Investigación le deberán seguir la publicación de obras, resultado y evidencia del trabajo colaborativo interinstitucional regional entre pares académicos, propiciado a través del establecimiento de redes entre los cuerpos colegiados de las instituciones miembros del Consejo, para fortalecer sus capacidades de investigación y promover su desarrollo a través de la producción científica colegiada de alto nivel, en específicas Líneas de Generación, Innovación y Aplicación del Conocimiento.

Los Encuentros Nacionales de Comunicación deberán seguir siendo espacios dinámicos y plurales de reflexión conjunta entre investigadores, 
docentes y estudiantes de Comunicación, de intercambio de experiencias, de enriquecimiento mutuo con propuestas innovadoras de investigación, de enseñanza, de aprendizaje, de vinculación con las exigencias del campo profesional de nuestros egresados. Además, todas las vocalías han programado la realización anual de encuentros regionales, ya sea seminarios teórico-metodológicos para promover el desarrollo de las competencias docentes, cursos-talleres para discusión y análisis de las áreas temáticas que competen a nuestro campo de estudio, yforos académicos entre docentes y estudiantes.

Existen dos modalidades nuevas de trabajo colaborativo en las que queremos incursionar: la creación de cátedras de comunicación y el auspicio para la creación de programas académicos de excelencia a nivel posgrado, en forma interinstitucional.

Respecto a las cátedras, espacios de cooperación académica y científica para consolidar la formación y la investigación de alto nivel, consideramos que el Coneicc está en la posibilidad de incursionar en dichos ámbitos para adecuarse a las expectativas de las instituciones de educación superior que la conforman, y colocarse a la vanguardia del campo de la comunicación.

Por otro lado, la oferta de posgrados de calidad "incorpora la generación y aplicación del conocimiento como un recurso para el desarrollo de la sociedad, así como la atención de sus necesidades, contribuyendo a consolidar con mayor autonomía y competitividad el crecimiento y el desarrollo sustentable del país". ${ }^{1}$ Por lo tanto, el auspicio por parte del Coneicc de programas académicos de excelencia a nivel posgrado, en forma interinstitucional, ${ }^{2}$ uniendo sinérgicamente y aprovechando los recursos con que se cuenta al interior del Consejo, le permitirá dar pasos muy grandes en el cumplimiento de uno de sus objetivos, que es la promoción de la excelencia en los programas académicos de comunicación a nivel superior y el fomento de la cultura de evaluación y de calidad académica a nivel nacional e internacional.

Hemos presentado a grandes rasgos el proyecto para tres años de trabajo, de conexión e interacción, en orden con la conformación de un tejido cohesionador, de un discurso grupal construido por todos los integrantes del Coneicc, abierto a la aceptación y reconocimiento de las alteridades que lo constituyen, y a la comprensión común de los problemas sociales, técnicos y educativos de la realidad nacional para tratar de

\footnotetext{
${ }^{1}$ Consultado en la siguiente página web: www.conacyt.mx/calidad/Becas_Programas PosgradosNacionalesCalidad.html

${ }^{2}$ Proyectos que respondan a los parámetros de calidad para ser incluidos en el Programa Nacional de Posgrados de Calidad (PNPC) del Conacyt.
} 
encontrar soluciones a los mismos, razón última del quehacer académico de la comunicación.

\section{LA OBRA}

La edición XVI del Anuario de Investigación de la Comunicación Coneicc se compone de siete textos de diversa índole y temática, ordenados alfabéticamente por autor, quienes provienen tanto de instituciones públicas como privadas. A este conjunto de trabajos se agrega una importante aportación: la reseña de las tesis ganadoras (tres de licenciatura y una de maestría) del Premio Nacional Coneicc 2008, y cuya incorporación al Anuario tiene como finalidad manifestar el interés por divulgar el esfuerzo intelectual de jóvenes investigadores cuyo trabajo recepcional fue calificado por el Comité de Evaluación de Tesis del Coneicc, como merecedores de ser premiados.

El primer trabajo proviene de un grupo de trabajo encabezado por Julieta Carabaza González, profesora investigadora de la Facultad de Ciencias de la Comunicación de la Universidad Autónoma de Coahuila, y tres estudiantes de la misma institución: Omar Muñoz Cardona, Pablo Olmos Peña y Tania Ortiz Silva. El tema es por demás interesante, pues aborda una problemática contemporánea que lleva por título "Consumo de televisión y actitudes ambientales en universitarios". Este es un ejercicio de investigación cuyos resultados presentados son parte de una primera etapa de un proyecto mayor que busca, a partir del entendimiento del papel que juegan los medios de comunicación en la construcción de la cultura ambiental, proponer algunas directrices para la elaboración de estrategias de comunicación ambiental. Entre los hallazgos más importantes se encuentra que todos los estudiantes de dicha universidad están concientes de los problemas ambientales de la ciudad; sin embargo, no se evidencia compromiso para la acción.

Le sigue el texto de Blanca Chong, Gabriela Aguilar Ramos, Alfredo Morales Pérez y José Luz Ornelas López, todos ellos académicos de la Universidad Autónoma de Coahuila, que presentan un trabajo denominado "Consumo de mensajes audiovisuales por género, en cuatro ciudades del noreste de México". El documento muestra los desequilibrios en la oferta de mensajes audiovisuales entre países y sus consecuencias para la cultura y la identidad de las naciones menos desarrolladas; esta es una temática que ha estado presente en las últimas décadas entre los estudiosos de la comunicación. En este artículo se exponen algunos de los hallazgos obtenidos en las ciudades de Monterrey, Saltillo, Torreón y Reynosa, en la investigación "Consumo de medios audiovisuales en el noreste de Méxi- 
co", como parte del proyecto que desarrolla la Vocalía Noreste del Coneicc. Los resultados aquí reportados confirman la hipótesis de la proximidad cultural. Las audiencias de la región noreste del país, a pesar de su cercanía con los Estados Unidos, mantienen una fuerte exposición a la programación de la televisión nacional, aunque con diferencia en las cuatro ciudades, basadas en la mediación de género.

Los autores del tercer trabajo que presenta el Anuario son Martín Echeverría Victoria y Maricarmen Garay Hernández, profesores de la Universidad Anáhuac Mayab, y lleva por título "Rechazo sin evidencia: prejuicio político entre jóvenes meridanos". Este artículo refiere a un tema muy a propósito de los tiempos políticos que en este 2009 vivió México, relativos a los procesos electorales. Dicho texto muestra una preocupación fundamental para la política mexicana: el aparente desinterés y apatía de los jóvenes hacia la participación política, sus instituciones y acontecimientos. Para abordar estos fenómenos, parte de la socialización política se concentra en las representaciones que los jóvenes tienen sobre el gobierno. Recurriendo a un diseño cualitativo consistente en grupos focales y entrevistas profundas, sus hallazgos indican la reproducción de actitudes y valores de la cultura política mexicana en los jóvenes; una representación personalizada y apolítica del gobierno; y el papel de la familia, uno de los pocos vínculos entre los jóvenes y el sistema político.

Javier Esteinou, profesor de la Universidad Autónoma MetropolitanaXochimilco, presenta en el cuarto trabajo la problemática que enfrenta el actual modelo económico nacional, y para ello aporta un texto intitulado "La función del modelo de 'comunicación casino' en la crisis del sistema neoliberal contemporáneo". En él refiere que el tipo de "economía casino" que originó la crisis económica contemporánea en el año 2008, funcionó gracias a que construyó un modelo de "comunicación casino" que promovió los valores bárbaros del capitalismo salvaje y fomentó la compra de "inversiones basura", derivados financieros de alto riesgo y productos bursátiles tóxicos paralelos que durante varios años generaron dicho modelo especulador. Por ello, la verdadera corrección de tal hundimiento económico no se ordenará con la simple inversión financiera de los Estados nacionales, sino que se solucionará con el saneamiento profundo de las raíces ético-morales que lo originaron.

El siguiente trabajo aborda un campo por demás interesante como fructífero en términos de tratamiento académico. El cine es el objeto de estudio de José Samuel Martínez López y Gibrán Larrauri Olguín, profesores de la Universidad Iberoamericana, Ciudad de México. Su texto lleva por nombre "Cine y psicoanálisis. Un ensayo sobre sus convergencias y bifurcaciones". Al respecto, dichos autores refieren que ambos campos 
tienen un origen decimonónico, y que tanto uno como el otro se deben a la propulsión científica y tecnológica, que es factor de gestación del discurso de la modernidad, discurso que propone un avance progresivo y predecible hacia estados de mayor concordancia entre el sujeto y su mundo. Pero si bien el cine y el psicoanálisis son producto cultural de la modernidad, no por ello se podría indicar que son completamente afines a ella, o que incluso van en la misma dirección.

La televisión como nodo central de la cotidianeidad mediática, permite su escudriñamiento desde diferentes vertientes por parte de los investigadores de la comunicación. El sexto trabajo del Anuario contiene el documento de José Antonio Meyer Rodríguez, de la Universidad Popular Autónoma del Estado de Puebla, intitulado "Del control de las redes al reto de los contenidos: Televisa ante el nuevo proyecto de televisión hispana en Estados Unidos". Al respecto, el autor confecciona un amplio y bien documentado análisis sobre la televisión hispana estadounidense, y la forma como los diferentes actores se adaptan a las cambiantes condiciones de mercado, además de la importancia creciente de Univisión en la industria comunicativa global y las consecuencias de su reestructuración en el proyecto de expansión de Televisa en Estados Unidos.

El último texto de esta parte de trabajos del Anuario es el de Ma. Elena Zermeño Espinosa, de la Universidad Autónoma de Baja California, denominado "Una intervención comunitaria desde la comunicación para la salud", en el cual presenta un estudio e intervención comunitaria en esa relación todavía en proceso de consolidación entre disciplinas, en ocasiones tan disímiles, como lo son la comunicación y aquellas relacionadas con la salud. La autora describe ese paso que busca la interacción entre los diferentes sectores, instituciones e integrantes que conforman la sociedad; además, la comunicación facilita la vinculación entre las distintas disciplinas que contribuyen a la promoción para la salud integral de las personas, como la medicina, enfermería, psicología, educación, sociología, derecho, psiquiatría, deportes, artes, la propia comunicación, y otras.

Finalmente, el Anuario de Investigación de Comunicación Coneicc concluye cumpliendo una de las tareas más importantes dentro de sus objetivos: dar oportunidad de divulgar la obra científica de los jóvenes investigadores de las ciencias de la comunicación. Dicho objetivo se logra con la publicación de los resúmenes de las tesis ganadores del Premio Nacional Coneicc 2008, los cuales, al ser seleccionados como los mejores tres lugares en la categoría de tesis de licenciatura y el mejor de la categoría de maestría por el Comité de Evaluación de Tesis del Coneicc, se hicieron merecedores de esta distinción dentro de la edición XVI del Anuario.

En la categoría de licenciatura, el primer lugar lo obtuvo Roberto Ignacio Alonso Muñoz, con el texto "Ley Televisa: poder mediático al 
desnudo", cuyo director de tesis fue Rafael G. Hernández García Cano, ambos de la Universidad Iberoamericana, Puebla. La tesis documenta y demuestra la magnitud de un poder privado con repercusiones adversas en el diseño de un país democrático. La intuición de la que se parte para este propósito es que los medios, y de manera más específica, la televisión, representa un poder fáctico en el espacio público mexicano, sin estar sujeto a las formas tradicionales del poder político para ejercerlo. El autor plantea que tras indagar en las inconstitucionalidades de la Ley Televisa, se descifra que la lógica alrededor de este paquete de reformas giró en tres ejes, a saber: la consolidación del dominio de los actuales concesionarios de la radiodifusión sobre el espectro radioeléctrico como si se tratara de propietarios, excluyendo y evitando así la entrada de nuevos competidores y/o de otras formas de comunicación social como los medios públicos y los medios comunitarios; la expansión del ámbito de acción de estos concesionarios al mercado de las telecomunicaciones, sin que este pase automático para ofrecer nuevos servicios bajo la misma banda de frecuencia supusiera una contraprestación económica al Estado; y por último, su protección con un órgano regulador aislado del poder ejecutivo y débil frente a los entes regulados, al no contar con la posibilidad jurídica de negar la renovación de las concesiones al vencer su plazo.

El segundo lugar de la categoría de licenciatura correspondió al texto "La transculturación de una familia de migrantes mixtecos en la colonia Ferrocarril a través de la comunicación intercultural (estudio de caso)", cuya tesista es María del Carmen Padilla Arreguí, quien tuvo como asesora de tesis a Verónica Victorica Pérez, ambas de la Universidad del Valle de Atemajac, Jalisco. El trabajo es un estudio de caso enfocado en una familia de migrantes mixtecos de la colonia Ferrocarril, sumergida en la urbe, en la zona metropolitana de Guadalajara, orientado a describir la transculturación de este grupo étnico, con un enfoque de comunicación intercultural. Como parte de esta investigación, las categorías establecidas como ejes del cambio cultural fueron: festividades religiosas, red de relaciones, lengua, artesanías e indumentaria; cada categoría está interrelacionada con procesos de comunicación y cultura. El contexto social forma parte importante del trabajo, de forma que los sucesos que anteceden y los procesos sociales que lo contextualizan enriquecen la investigación, logrando un mayor argumento basado en tres tópicos centrales: indigenismo, cultura y migración, los cuales le dan mayor amplitud y comprensión al estudio.

El tercer lugar de esta categoría lo obtuvo María de Lourdes González Franco, con su tesis "Queer as folk: punto de ruptura del estereotipo homosexual en el contenido de los medios audiovisuales", cuyo direc- 
tor de tesis fue Jaime Raymundo Ramírez Ortiz, por la Universidad del Tepeyac. Queer as folk es una serie televisiva cuyo origen proviene de Inglaterra, y que posteriormente se produjo en los Estados Unidos; en ella se muestran personajes homosexuales más cercanos al pluralismo de la comunidad homosexual y su quehacer como unidad social e individual dentro de la sociedad actual, alejándose de los estereotipos que estigmatizan a este colectivo social. Así, el objeto de estudio de este trabajo es el estereotipo que del homosexual se ha manejado en los medios audiovisuales. El procedimiento de esta tesis implicó una investigación documental sobre los tópicos relacionados con el problema, para luego aplicar el método de análisis de contenido, extrayendo temáticas abordadas en la serie, al igual que la descripción detallada de los personajes de la trama, a la vez que comparar lo expuesto en Queer as folk con algunos casos representativos dentro del cine y la televisión, tanto de producción extranjera como nacional.

En la categoría de mejor tesis de maestría, el trabajo premiado fue de Rosalía Orozco Murillo, de la Universidad de Guadalajara, denominado "Relaciones prensa-gobierno en Tepic: hacia la caracterización de prácticas predominantes en el periodismo local de México"; su asesor de tesis fue María Elena Hernández Ramírez. Esta tesis aborda, desde una perspectiva histórico-estructural, el complejo fenómeno de las relaciones prensa-gobierno en México. A través de una estrategia metodológica de tipo cualitativa, la investigadora caracteriza y describe las prácticas, conductas y costumbres típicas y atípicas que se han dado históricamente en la relación social que establecen los periodistas y los funcionarios públicos en el contexto particular de Tepic. La autora asegura que algunas de las características que prevalecen en el trato entre la prensa y el gobierno en Tepic remiten a un modelo de relación típico de un régimen autoritario, aunque otras características permiten ubicar la relación entre esos dos actores en un periodo de transición democrática.

En una sucinta síntesis, la edición XVI del Anuario de Investigación de Comunicación Coneicc da espacio a una diversidad de enfoques y abordajes del campo de la comunicación y sus vínculos con otras disciplinas con las cuales comparte preocupaciones científicas, pero también la necesidad de dar respuesta a problemas ingentes. Así, se analiza tanto la tradición del estudio de los medios como la televisión, la prensa o el cine, como también, desde una perspectiva más amplia, la relación entre comunicación y medioambiente, salud y el psicoanálisis. Además, en este trabajo son objeto de estudio tanto migrantes mixtecos como jóvenes o familias en comunidades urbanas, homosexuales o estudiantes universitarios. Asimismo, se presentan diversos métodos y técnicas de recolección de datos, 
producto de los afanes de los investigadores por esclarecer su problema de investigación. De igual manera, se encuentran representados en este Anuario trabajos de académicos de diversas instituciones, tanto públicas como privadas, y cuya trayectoria en el campo data de varios ayeres, así como de otros tantos que se inician en esta actividad con el entusiasmo por ver publicados sus primeros ejercicios intelectuales.

Vaya a todos el agradecimiento por su contribución a la edición XVI del Anuario de Investigación de Comunicación Coneicc, por parte de quien le correspondió coordinar este esfuerzo colectivo que da cuenta fiel de la capacidad, talento y productividad de los académicos mexicanos.

Mexicali, Baja California; octubre de 2009. 